\title{
The 1980 pressure response and flank failure of Mount St. Helens (USA) inferred from seismic scaling exponents
}

\author{
S. Vinciguerra ${ }^{\mathrm{a}, *}$, D. Elsworth ${ }^{\mathrm{b}}, \mathrm{S}$. Malone $^{\mathrm{c}}$ \\ ${ }^{a}$ Osservatorio Vesuviano- Istituto Nazionale di Geofisica e Vulcanologia, Naples, Italy \\ ${ }^{\mathrm{b}}$ Department of Energy and Geo-Environmental Engineering, 231 Hosler Building, University Park, PA 16802, USA \\ ${ }^{\mathrm{c}}$ Earth and Space Science, University of Washington, 310 Condon Hall, Box351310, Seattle, WA 98185, USA
}

Accepted 16 November 2004

\begin{abstract}
The cataclysmic 18 May 1980 eruption at Mount St. Helens was preceded by intense seismic activity marking the mechanical response of the volcanic edifice to interior pressurisation. This seismicity is analysed to yield the temporal change in the seismic scaling exponent, $D$, inferred from the seismic $b$-value, that in-turn is related to the seismic moment of an earthquake. Time evolution of $D$ preceding the eruption onset reveals: (1) a major decrease in $D$ occurring over only a few days at the end of March; (2) a steady but stepped decrease in $D$ (steps $\sim 5-10$ days) occurring from the end of March to early May; (3) a sharp decrease in $D$ in early May; and (4) steady low values of $D$ occurring 2-3 days before the eruption onset. This response is interpreted as major ruptures, formed at the end of March, arresting and participating in, but not triggering the ultimate failure of the flank. Rather, the rate of interior fracturing slowed in the 2 months preceding the 18 May 1980 major blast, and the triggering of failure is consistent with interior gas overpressurisation. The occurrence of two swarms of low frequency seismic events and the high values of the harmonic tremor indicate the action of interior pressurisation on a cycle of $\sim 20-25$ days. Solutions are applied to represent the harmonic interior pressurisation of the edifice by gas exsolving from the volcano core. The transient radial migration of overpressured gas may reduce the apparent strength of the edifice, and ultimately trigger failure of the flank. Importantly, this mechanism is capable of triggering flank failure both after multiple core pressurisation cycles have been sustained, and as core pressures are low and diminishing — and may be a minimum. These twin attributes are both apparent in the seismic record for Mount St. Helens, used as a proxy for the unrecorded timing and magnitude of gas pressurisation at the volcano core.
\end{abstract}

(C) 2004 Elsevier B.V. All rights reserved.

Keywords: seismicity; scaling exponents; harmonic inflation; pressurisation mechanics; rupture dimension

\footnotetext{
* Corresponding author. Tel.: +39 816108443 ; fax: +39 816108351 .

E-mail address: vinciguerra@ov.ingv.it (S. Vinciguerra).
} 


\section{Introduction: the 1980 eruption at Mount St. Helens}

Mount St. Helens is a dacitic stratovolcano, originally $2951 \mathrm{~m}$ in height in southwest Washington State, USA. While Mount St. Helens is one of the youngest of the Cascade volcanoes, it is also one of the most active, with several major eruptions and numerous minor eruptions recorded over the past few thousand years. The climactic phase of its most recent eruption, on 18 May 1980, was initiated with the decapitation of the mountain by a giant landslide. This initiated on the flank of the volcano, removed the top $400 \mathrm{~m}$ to the summit, and mobilised a total rock volume of $2.4 \mathrm{~km}^{3}$. The landslide triggered a lateral blast which destroyed more than $600 \mathrm{~km}^{2}$ of the surrounding forest to the north of the volcano. The blast was followed by a plinian eruption lasting almost $9 \mathrm{~h}$ and depositing about $0.24 \mathrm{~km}^{3}$ of pyroclastic flows and ash on the north flanks (Christiansen and Peterson, 1981).

The precursors to the eruption were among the most intense and dramatic of any well-documented volcanic episode. In addition to a very intense earthquake sequence, the volcano experienced numerous small phreatic steam explosions and very rapid ground deformation during the 2 months immediately preceding the cataclysmic eruption. All these precursory signals were recognised at the time and were used to evaluate the increasing hazard. Neither the exact time nor the ultimate size of the eruption was predicted. However, significant areas around the volcano were closed to public access thereby reducing casualties from the ultimate eruption. Seismicity and ground deformation data show no obvious trends nor changes that could be used, even a posteriori, to predict the time of the eruption. Key to this observation is that collapse of the flank occurred on a downturn in seismicity. Where seismicity is taken as a direct proxy for interior magma or gas pressurisation, this suggests that failure occurred as the interior forces driving failure had peaked, and diminished. We explain this observation through the application of a consistent model for fracture growth and concurrent interior gas pressurisation. In particular, this model replicates the enigmatic phase-delay between peak pressurisation, and the initiation of runaway failure. This model is described in the following, in particular in relation to the May 18th 1980 eruption of Mount St. Helens.

\section{Seismicity}

Routine seismic monitoring had been conducted since the installation of the first station by the University of Washington in 1972. In March 1980 only one seismic station was operating near Mount St. Helens, with the next nearest station $50 \mathrm{~km}$ to the north of the volcano. After the first recognisable precursory earthquake occurred on 20 March 1980 additional seismic stations were installed on and near the volcano. Data from some of these stations were transmitted in real-time to the University of Washington where they were recorded in a data acquisition and processing system. Data from other stations were recorded locally on magnetic tapes for later play-back and analysis. Only a few hundred of the more than 4000 recorded earthquakes were timed and located, because of the time-consuming task and difficulties in processing data. Hypocenter determinations for earthquakes had varying quality depending on the number of operating stations near the volcano and the signal quality at those stations. In all cases, those earthquakes which could be located were clustered in a small volume, and at shallow depth, directly under the north flank of the mountain.

While only a small fraction of the recorded earthquakes could be located, a fairly detailed and complete listing of the number of events is available. These events are culled from manual reviews of the visual seismograms. Records from stations located in the western and eastern flank of the volcano were routinely reviewed for seismic events coming from Mount St. Helens. The time, type, and size of each event were noted in a list. Events were classified based on their seismograms into type-L (low-frequency or LP (long period) type events), type-E (shallow volcano-tectonic type events with medium frequency), and type- $\mathrm{H}$ (high-frequency deeper volcano-tectonic events). Even though few of the $\sim 4000$ precursory events were located, their character and locations indicate that all of them originated under the volcano in response to the rising magma. The type- $\mathrm{L}$ and type-E events are shallow, either within or just below the volcano edifice, while the type-H events are 
deeper and likely associated with the crustal magma chamber or conduit system (Scandone and Malone, 1985). Major features of the precursory earthquake swarm preceding the 18 May 1980 eruption include:

- a gradual increase of very small volcanic earthquakes starting in mid-March;

- a magnitude 4.2 earthquake on March 20;

- a significant increase in the number and size of events after March 23;

- an almost saturated record for nearby stations on March 26 and 27;

- a gradual decrease in the daily number of earthquakes after March 27; and

- xa magnitude 5.1 earthquake triggering the landslide and the lateral blast, on the morning of 18 th May 1998.

While the number of earthquakes decreased in April and early May, the average size of the events increased. There were also several periods of very intense volcanic tremor during April, some of which could be recorded on seismograph stations $200 \mathrm{~km}$ from the mountain (Endo et al., 1981). The overall distribution of seismic events during January to May 1980 is shown in Fig. 1.

The first clear evidence of volcanic activity was a steam explosion on March 27 at the height of a seismic swarm (Fig. 1). A small crater formed at the top of the volcano that enlarged over the following several weeks as additional phreatic steam explosions occurred. Either starting with the first explosion or shortly thereafter, cracks formed across the summit region and the north flank of the volcano began to expand. By mid-April, when deformation monitoring by the USGS became routine, the deformation rate was a constant at $1.5 \mathrm{~m} /$ day and continued with no significant change until the eruption on 18 May (Lipman et al., 1981).

These observations of ground deformation, the ultimate breeching of the flank by phreatic eruptions, and escalation to the final collapse and lateral blast on the north flank, may be correlated with the seismic data. Duration magnitude for all recorded events was estimated by the equation $M_{\mathrm{d}}=-2.66+2.74 \times \log$ (duration) (Malone, 1990). A test for completeness of the seismic data set, using the procedure of Tinti and Mulargia (1985), shows that the data set may be considered complete for $M_{\mathrm{d}} \geq 2.0$ over the entire period analysed (January through June, 1980).

The daily average magnitude of seismic events for the period March through June 1980 is shown in Fig. 2. This daily average magnitude shows a sharp increase at the end of March related to the occurrence of energetic seismic swarms. This rapid increase markedly slowed in early April, synchronous with the decrease in seismic activity, but again increased slightly beginning in early May until the time of the eruption on May 18th. The majority of the recorded seismicity is attributed to volcano-tectonic events. These earthquakes are associated with the structural response of the volcanic edifice to stresses induced by

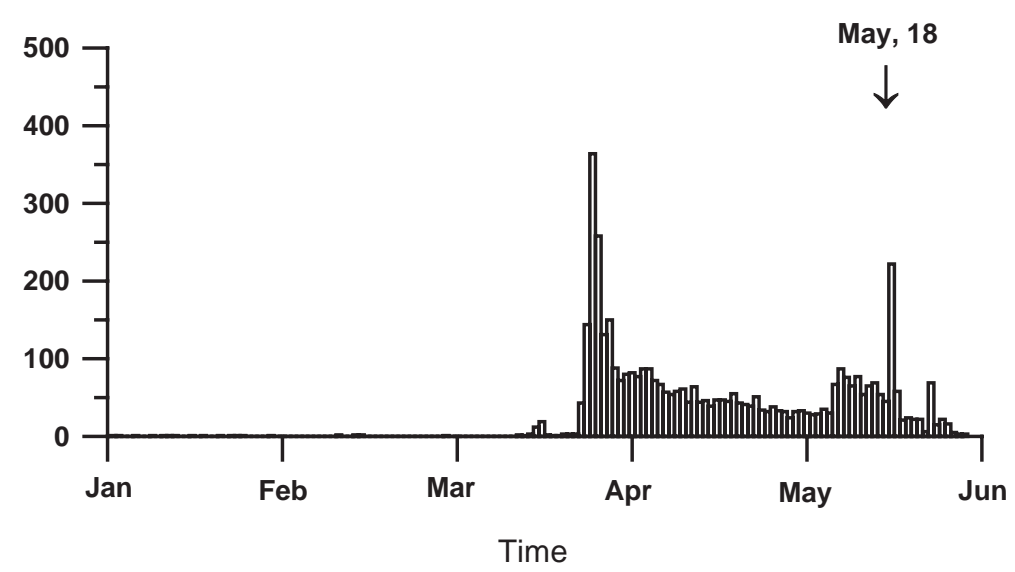

Fig. 1. Daily frequency distribution at Mount St. Helens during January to May 1980. 


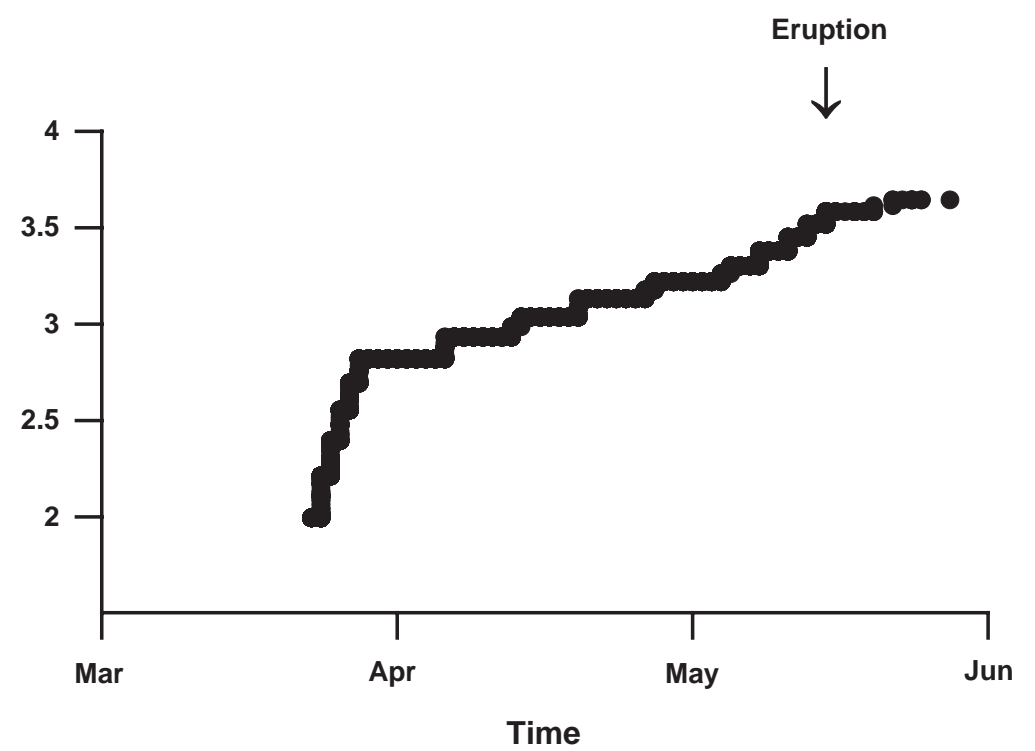

Fig. 2. The daily average duration magnitude vs. time during March to May 1980. Earthquakes with $M_{\mathrm{d}} \geq 2.0$ occur before the end of March.

the injection of new magma (Shaw, 1980; Takeo, 1983) or to pressure changes within the existing magma body. Correspondingly, these are useful indicators of brittle fracture, and may be used to map evolving (effective) stress concentrations distributed throughout the large rock volume surrounding the growth of the edifice, the magma conduit, and the cryptodome.

Low-frequency events and tremor implicate the presence and active participation of fluids in the inflation process. These pressure fluctuations may result from the unsteady mass transport and/or the thermodynamics of the fluid, and may reduce the strength of the surrounding rock mass and promote failure. Plausible mechanisms of failure include thermal cracking, hydraulic fracturing, loss of block interlock, and through hydrothermal weakening (Elsworth and Voight, 2001). The active participation of fluids in the eruption of May 18, 1980 is implicated in the cyclic intensities of low-frequency events and harmonic tremor documented in Fig. 3. Two intense swarms of low-frequency events occurred in midApril and early May. Mid-to-high values of harmonic tremor preceded and accompanied the swarms. This pattern can be related to two main cycles of fluid pressurisation. These occur with a recurrence period of 20-25 days, the first following the intense volcanotectonic seismic activity of end of March and the

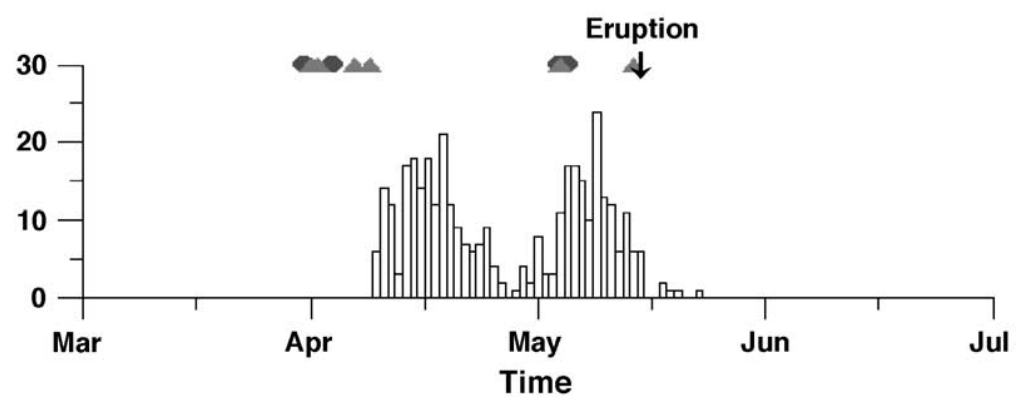

Fig. 3. Daily distribution of low-frequency events. Triangles mark very strong harmonic tremor and circles mark moderately strong (all stations around Mount St. Helens) harmonic tremor. Arrow marks eruption onset. 
second preceding the mid-May earthquake swarms that immediately precede the eruption.

In the following sections we first estimate the mechanical response and the evolution of brittle damage that is consistent with the observed seismicity, and suggest how runaway failure may develop, even as the intensity and rate of damage subsides. The evolution of damage within the edifice is marked by the time evolution of the power law exponent $D$ of the fracture distribution - this is inferred from the magnitude and recurrence-frequency of the volcano-tectonic events. These data are then applied with a consistent model for the interior inflation of the edifice, capable of explaining the phase-delay between peak edifice pressurisation and the delayed timing of collapse.

\section{The power law exponent $D$ of the crack length distribution}

\subsection{Significance of the seismic scaling exponent $D$}

If earthquake sources can be regarded as simple scale-invariant dislocations of finite extent (Kanamori and Anderson, 1975) the power-law exponent $D$ is proportional to the seismic $b$-value (Aki, 1981) and can therefore be inferred either from earthquake or acoustic emission data (Meredith et al., 1990; Main, 1991) via:

$D=3 b / C$

where $C$ is a scaling parameter between magnitude and the common logarithm of the seismic moment. $C$ is equal to $3 / 2$ for earthquakes of similar corner frequency to the natural period of the seismometer (Kanamori and Anderson, 1975), so that $D=2 b . D$ is not strictly a fractal dimension since it does not provide any positional information (Mandelbrot, 1982), but it reflects different aspects of scale invariance, being an expression of the self-similarity of seismic energy (Turcotte, 1989; Main, 1991). $D$ is related to the area and the displacement across the rupture and measures the relative proportion of large and small fractures or cracks (Turcotte, 1989; Main, $1992)$ that generate seismic events. High values of $D$ record the relative prevalence of the generation of small fractures, while low $D$ values mark the prevalent formation of larger fractures.
Correspondingly, the time evolution of $D$ may be a good indicator of the mechanisms promoting damage within a rock body, in this instance used as a proxy for the processes preceding or accompanying volcanic eruptions (Vinciguerra, 1999; 2002). The $b$ value of the Gutenberg and Richter (1956) relationship has been estimated using the maximum likelihood method (Aki, 1965):

$b=\frac{\log _{10} e}{<m>-m_{0}}$

where $m_{0}$ is the threshold magnitude for complete reporting of earthquakes magnitudes. The error at $95 \%$ of the confidence limit (Aki, 1965) is:

$\Delta b=\frac{1.96 b}{n^{1 / 2}}$

where $n$ is the total number of earthquakes in the particular data set considered. Correspondingly, the seismic scaling exponent and the $b$-value are usefully related, as, $\Delta D=(3 / 2) \Delta b$. Generally $b \sim 1$, but its value is often greater in volcanic areas, where $b$ can reach values close to 2 (Minakami, 1990; Wyss et al., 1997). We use this relationship to estimate the change in the seismic scaling exponent immediately preceding the 1980 eruption of Mount St. Helens.

\subsection{Time evolution of the seismic scaling exponent $D$}

The temporal evolution of the seismic scaling exponent is evaluated for the 4-month window immediately preceding the eruption of Mount St. Helens on May 18, 1980. We use a moving window of 60 events, with a 1 event overlap. The limited overlap highlights changes in the temporal variations in $D$, by avoiding excessive cumulative averaging. The value calculated inside each window is assigned to the time occurrence of the last event.

The resulting magnitude of the seismic scaling exponent, $D$, shows significant temporal variation (Fig. 4a,b) both in the medium-term (of the order of several days) and the short-term (of the order of a few days). Integrating these results with the geophysical evidence reveals the following patterns:

1) The scaling exponent $D$ decreases rapidly (Fig. 4a) at the end of March; the drop is from $D \sim 5$ to $\sim 1$, 

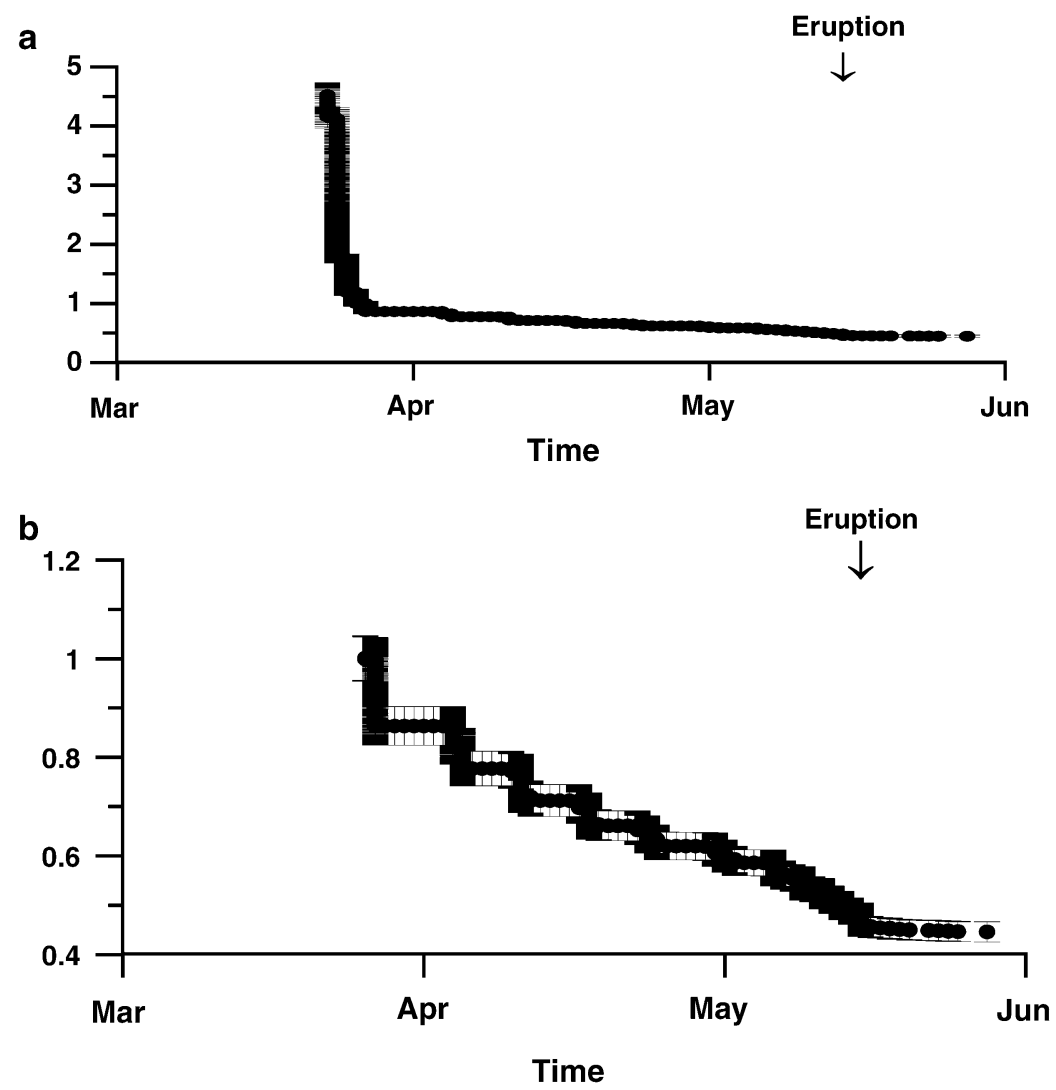

Fig. 4. Time evolution of seismic scaling exponent $D$ during 1980 (a) over January-May; (b) end of March-May.

and occurs over only a few days. This pattern is consistent with the rapid and energetic increase in seismic activity that occurs over the 5 days immediately following the magnitude 4.2 earthquake (20 March), and is confirmed by the concurrent sharp increase in the daily average magnitude (Fig. 2). These contributing events were located within a limited volume directly beneath the volcano, and at a shallow depth. This episode was closely followed by phreatic eruptions and concurrent continuous deformation of the north flank, beginning on March 27 (Malone, 1990). The deformation was manifest as brittle surface ruptures, resulting from the response of the flank to the presumed rapid injection of magma and associated gases. However, despite the intensity of the surface-breeching deformation, ultimate failure of the flank stalled.
2) $D$ decreases (Fig. $4 \mathrm{~b}$ ) to a near constant rate-ofchange. The magnitude decreases from $D \sim 1$ to $D \sim 0.3$ over about 45 days, in steps of $\sim 5-10$ days. This occurs from the end of March to early May. This pattern indicates a reduction in the rate of new fracture production, and thereby in the rate of flank weakening. In turn this delays the ultimate failure of the edifice, allowing other mechanisms to become dominant. For Mount St Helens, this dominant mechanism is through interior pressurisation, as discussed later. These trends are supported by the evidence that the average magnitude of the seismicity increased (Fig. 2), despite the apparent decrease in the daily frequency of seismicity (Fig. 1). This supports the view that large fractures that formed at the end of March continued to grow slowly and constantly. During this period the rate of vertical deformation was a remarkably steady $1.4 \mathrm{~m}$ per day, although the 
variability in time sampling makes it difficult to correlate changes in line length and earthquakes (Lipman et al., 1981; Malone, 1990). These observations are consistent with a model of slow fracture extension induced by loading and magma overpressure (Kilburn and Voight, 1998; Kilburn, 2003), in which hydrofractures extend by the slow growth and coalescence of smaller cracks (Anderson and Grew, 1977; Kilburn and Voight, 1998; Main, 1999; Kilburn, 2003). Large hydrofractures are then temporarily 'locked' for 5 to 10 days (steady $D$ values), until a given threshold of damage is reached and overcome at the fracture tip, as evident both from field (Gudmundsson et al., 2002) and experimental (Lockner et al., 1991) observations. A plausible explanation is that smaller but non-interconnected ruptures evolve in the intermediate period (no $D$ variation) and that these slowly coalesce, and finally allow the larger ruptures to propagate (step decrease in $D$ ). This process repeats, and results in the intermittent propagation of ruptures, observed in the step-like decrease in the scaling exponent (Fig. 4b). The seismic scaling exponent $D$ provides the evidence in support of such a multiscaling process (Kilburn, 2003).

3) The scaling exponent, $D$ exhibits a sharp decrease (Fig. 4b) in early May; the exponent reduces from $\mathrm{D} \sim 0.3$ to $\sim 0.2$ over a 7 -day period, concurrent with a slight increase in the earthquake rate (Fig. 1) and in the average earthquake magnitude (Fig. 2). This period includes large events, reaching $M=5$ (May 7), and correlates with the beginning of a new phase of vigorous phreatic eruptions (Malone, 1990). This period is interpreted as representing renewed crack growth, the widespread coalescence of discontinuous cracks into larger cracks, and the ultimate destruction of any prevailing temporary locking mechanism (Kilburn, 2003), leaving the system ready to accelerate to failure.

4) The final phase is represented by near steady but low values of the scaling exponent, as $D \sim 0.2$ (Fig. 4b) from about 5 May until the May 18 eruption. This is interpreted as the prevalence of an array of major cracks that form a kinematic pathway for the ensuing dynamical failure (Lockner et al., 1991; Main and Meredith, 1991); a failure that is predominantly driven not by continuing generation of new fractures, but by the reduction in strength of existing fractures by gas overpressurisation. We explore the potential for gas pressures to both trigger the ultimate collapse of the edifice, and to do this during a downturn in seismicity when assumed interior conduit gas pressures are diminishing.

\section{Pressurisation mechanics}

We explore the potential for the edifice to ultimately fail, not as a result of the generation of new fractures, but from the weakening of existing fractures by interior fluid pressurisation. The idealised case of a roughly hemispherical volcanic edifice of radius $b$, penetrated by a pressurised conduit, is considered first (Fig. 5). To aid the mathematical development, the connection between the edifice and the conduit is represented by a hemispherical cavity of radius $a$. In modelling magma and gas overpressures as a function of position and time, general transient solutions are developed to the harmonic pressurisa-
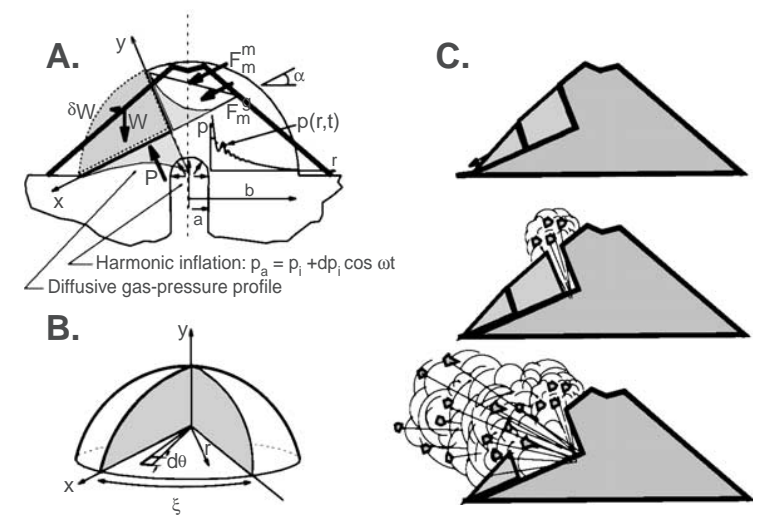

Fig. 5. Schematic section through a hemispherical edifice of external radius, $b$, overlying a gas-pressurised vertical conduit of radius, $a$. The conduit cap is harmonically pressurised as $p_{a}=p_{i}+d p_{i} \cos 2 \pi \omega t$, generating a gas uplift force, $P$, and rearscarp gas, $F_{m}^{g}$, or magma, $F_{m}^{m}$, disturbing forces acting on the wings of a failing block of weight, $W$, and included sector angle, $\xi$, resting on a detatchment surface inclined at angle $\alpha$ to the horizontal. Failure initiates on release of the block toe, with failure retrogressing to unroof the pressurised core, resulting in the potential for spontaneous disintegration or a directed explosion. 
tion of the dome core (Elsworth and Voight, 2001). The radial diffusion of gas overpressure, $p$, within the dome is controlled by the diffusion equation, as,

$\kappa\left(\frac{\partial^{2} p}{\partial r^{2}}+\frac{2}{r} \frac{\partial p}{\partial r}\right)=\frac{\partial p}{\partial t}$

where $\kappa$ is the gas or liquid diffusivity of a compressible fluid, $r$ is the radial ordinate, and $t$ is time (Carslaw and Jaeger, 1959).

Density and buoyant gas transport effects are ignored, as the dominant transport mechanism is by pressure fluctuations at the magma-conduit boundary. In this first-order analysis the permeability of the flank is assumed uniform, constant in time, and to be dominated by the presence of pervasive fractures - the unmeasured dependencies of permeability on gas saturation and stress level are incorporated in the selection of an average representative permeability. These second-order effects are minor in comparison to the role of interior gas pressurisation at levels approaching lithostatic stress (Voight and Elsworth, 2000). The models more closely approximate the case of endogenous dome growth, than exogenous dome growth associated with lobes that break out to the dome surface (Simmons et al., 2004), with associated release of gas.

The steady state solution for pressure-diffusive flow is controlled by the flank geometry, and transient and harmonic behaviour is additionally modulated by the fluid diffusion coefficient, $\kappa$ and the frequency of the pressurisation signal, $\omega$, discussed later. Destabilisation and collapse is ultimately controlled by the distributed form of this diffusing pressure signal, $\omega$ as embodied in gas pressures acting on a potentially unstable block, as illustrated in Fig. 5A.

Solutions are developed in the following for the transient harmonic non-inertial gas-pressurisation of a hemispherical volcano cap, and applied to define the effect on time-dependent instability. Of principal interest is the distribution of gas-pressures on planes of potential detatchment that may develop within the lava pile, demarking an unstable block that may be ultimately released from the flank and result in collapse.

\subsection{Instantaneous inflation}

The first step in developing the harmonic solution is to define the transient changes in interior gaspressure that result from the Heaviside application of initial pressure, $p_{i}(r=a)$, at the dome core, at time $t=0^{+}$. This internal pressurisation is superposed on the existing gas-pressure profile within the dome, and is typically orders of magnitude larger than the nondiffusive component (Voight et al., 1999). For gaspressure diffusion with radius, $r$, the solution of Eq. (4) for the boundary conditions of

$$
\begin{array}{lll}
p(r, t)=0 & a \leq r \leq b & t \leq 0^{-} \\
p(a, t)=p_{i} & r=a & t \geq 0^{+} \\
p(b, t)=0 & r=b & t \geq 0^{+}
\end{array}
$$

yields the distribution of pressures as (Elsworth and Voight, 2001)

$$
\begin{aligned}
p(r, t)= & p_{i} \frac{a}{r}\left[\frac{(b-r)}{(b-a)}\right. \\
& \left.-\sum_{n=1}^{\infty} \frac{2}{n \pi} \sin \left[n \pi \frac{(r-a)}{(b-a)}\right] e^{-n^{2} \pi^{2} \kappa t /(b-a)^{2}}\right]
\end{aligned}
$$

for a pressure of $p_{a}=p_{i}=$ constant at the dome core $(r=a)$. This solution contains two primary terms: a first term $p_{i} \frac{a}{r}\left(\frac{(b-r)}{(b-a)}\right)$ representing the steady pressure profile, and a second, $p_{i} \frac{a}{r}\left(\sum_{n=1}^{\infty} \frac{2}{n \pi} \sin \left[n \pi \frac{(r-a)}{(b-a)}\right]\right.$ $\left.e^{-n^{2} \pi^{2} \kappa t /(b-a)^{2}}\right)$ diminishing with time, which represents progress towards the steady state. This solution is the basic component to be used in defining harmonic behaviour.

\subsection{Harmonic inflation}

Where interior harmonic pressurisation of amplitude $d p_{i}$ and frequency, $\omega$, is superimposed on the Heaviside pressurisation, the interior pressure changes as $p_{a}=p_{i}+d p_{i} \cos (2 \omega \pi t)$ (see Eq. (5)). The resulting radially-decaying pressure profile will develop an harmonic overprint as illustrated in Fig. 5A. This radial distribution of pressure may be determined by 
applying Duhamel's theorem (e.g. Carslaw and Jaeger, 1959), as

$p(t)=\int_{0}^{t} \phi(\tau) \frac{\partial}{\partial t} F[t ;(t-\tau)] d \tau$

to represent behaviour of the linearized system by superposition. For this harmonic excitation, the forcing function is $\phi(t)=d p_{i} \cos (2 \pi \omega t)$, incorporating both the amplitude, $d p_{i}$ and forcing frequency, $\omega$, of the interior pressurisation. Substituting Eq. (8) for $F[t ;(t-t)]$ in Eq. (9), and using the forcing $\phi(t)=d p_{i} \cos (2 \pi \omega t)$, yields the harmonic solution for null mean pressurisation, $p_{i}=0$. Further adding the Heaviside solution of Eq. (8) results in a full solution for the instantaneous application of core pressure $p_{a}=p_{i}+d p_{i} \cos (2 \pi \omega t)$ against a null background pressure as (Elsworth and Voight, 2001)

$$
\begin{aligned}
p(r, t)= & p_{i} \frac{a}{r}\left\{\frac{(b-r)}{(b-a)}-\sum_{n=1}^{\infty} \frac{2}{n \pi} \sin \left[n \pi \frac{(r-a)}{(b-a)}\right]\right. \\
& \left.\times F_{D}\left[\omega_{D} t_{D} \pi_{D}, \frac{d p_{i}}{p_{i}}, \frac{a}{b}\right]\right\}
\end{aligned}
$$

with the component $F_{D}$ defined as

$$
\begin{aligned}
F_{D}= & \left(1+\pi_{D} \frac{d p_{i}}{p_{i}}\right) e^{-n^{2} \pi^{2} t_{D} / X}-\pi_{D} \frac{d p_{i}}{d p_{i}} \\
& \times\left[\cos \left(2 \pi \omega_{D} t_{D}\right)+\frac{2_{X \omega D}}{n^{2} \pi} \sin \left(2 \pi \omega_{D} t_{D}\right)\right]
\end{aligned}
$$

in terms of the ancillary variables

$$
\begin{aligned}
& \pi_{D}=\frac{1}{1+\frac{2 \chi \omega_{D}^{2}}{n^{2} \pi}} \\
& \omega_{D}=\frac{\omega b^{2}}{\kappa} \\
& t_{D}=\frac{\kappa t}{b^{2}}
\end{aligned}
$$

and $\chi=(1-(a / b))^{2}$. The accompanying coefficients, $\pi_{D}, \omega_{D}$, and $t_{D}$ are non-dimensional.

The most significant of these coefficients, in a physical sense, are those representing dimensionless inflation frequency, $\omega_{D}$, and diffusive time, $t_{D}$. Again, Eq. (11) comprises two components: the first representing steady behaviour $p_{i} \frac{a}{r}\left(\frac{(b-r)}{(b-a)}\right)$ and the second $p_{i} \frac{a}{r}\left(\sum_{n=1}^{\infty} \frac{2}{n \pi} \sin \left[n \pi \frac{(r-a)}{(b-a)}\right] F_{D}[\ldots]\right)$ the combined transient and harmonic effects, embodied in the term $F_{D}\left[\omega_{D}, t_{D}, \pi_{D}, \frac{d p_{i}}{p_{i}}, \frac{a}{b}\right]$ of Eq. (11). The square-bracketed terms represent the functional dependence of $F_{D}$.

In Eq. (11), the first term represents the transient behaviour and the second the harmonic response. As $t_{D} \rightarrow \infty$, the first exponential term collapses, leaving the steady harmonic portion. In this dynamical steady state there are two end-member behaviours of note. The first is at high inflation frequencies where $\omega_{D} \rightarrow \infty$ and $\pi_{D} \rightarrow 0$ and $F_{D} \rightarrow 0$; the resulting radial pressure distribution approaches that for inflation at a constant mean pressure of $p_{a}-p_{i}$, and inflation frequency has no influence. The second is at very low inflation frequencies, relative to the gasdiffusivity, where $\omega_{D} \rightarrow 0$, and consequently $\pi_{D} \rightarrow 1$ and the interior pressurisation becomes synchronous with the core pressurisation as $\left(d p_{i} / p_{i}\right) \cos \left(2 \pi \omega_{D} t_{D}\right)$. In this second mode, represented by very low inflation frequencies, the pressure profile will be at all times steady, and will monotonically approach the instantaneous core pressure, with decreasing radius; for $\omega_{D} \rightarrow 0$ the resulting inflation-synchronous pressure distribution is given everywhere as a multiple of the steady pressure distribution as $p_{i}=\left[p+d p_{i} \cos \left(2 \pi \omega_{D} t_{D}\right)\right] \frac{a}{r} \frac{(b-r)}{(b-a)}$.

\section{Pressure response and the failure mechanism at St. Helens}

We examine the response of a volcanic edifice to harmonic interior pressurisation. We define interior gas pressures acting on a potential slip surface, inclined at an angle $\alpha$ to the horizontal and cutting the toe of the edifice. The strength on this slip surface is cohesive and frictional-the frictional component of strength is controlled by effective stresses, which in turn are modulated by the distribution of evolving fluid pressures. Limit equilibrium stability is evaluated for a sector of the volcano, defined by the material above the potential slip surface, and confined within the sector, as illustrated in Fig. 5. Further details of the method are given in Elsworth and Voight (2001). Specifically, we examine the implications of harmonic interior pressurisation as the volcano is brought progressively towards failure, and the mechanisms that may be responsible for this process. This 
analysis is used to address the observations that failure occurs both after at least two cycles of seismic activity, taken as indicative of interior pressurisation, and that collapse ensues as seismicity and presumed pressurisation are diminishing. Significantly, failure occurs after a number of inflation cycles of similar intensity, and at the point of minimum seismic activity, taken as indicative of minimum internal gas pressure. Consequently, we address why failure occurred both following multiple successive and equally destructive inflation cycles, and at a time of low and declining interior pressurisation.

As identified in Eqs. (10) and (11), the magnitude of the uplift pressure is controlled by volcano geometry, the inclination of the potential detachment surface, and the crucial parameters of forcing frequency, $\omega$, and the relevant transport parameter of gas diffusivity, $\kappa$. The frictional component of strength, $\phi$, is conditioned by the temporally changing magnitudes of uplift pressures, that conditions the evolution to instability of the flank.

Broad ranges of the parameters controlling failure are available for the 1980 flank collapse and subsequent directed lateral blast at Mount St. Helens (Voight et al., 1983). The collapse geometry of the mountain is well defined, and represented by a summit $1000 \mathrm{~m}$ above the daylighting toe of a failure plane, dipping at $35^{\circ}$ (from the horizontal). The
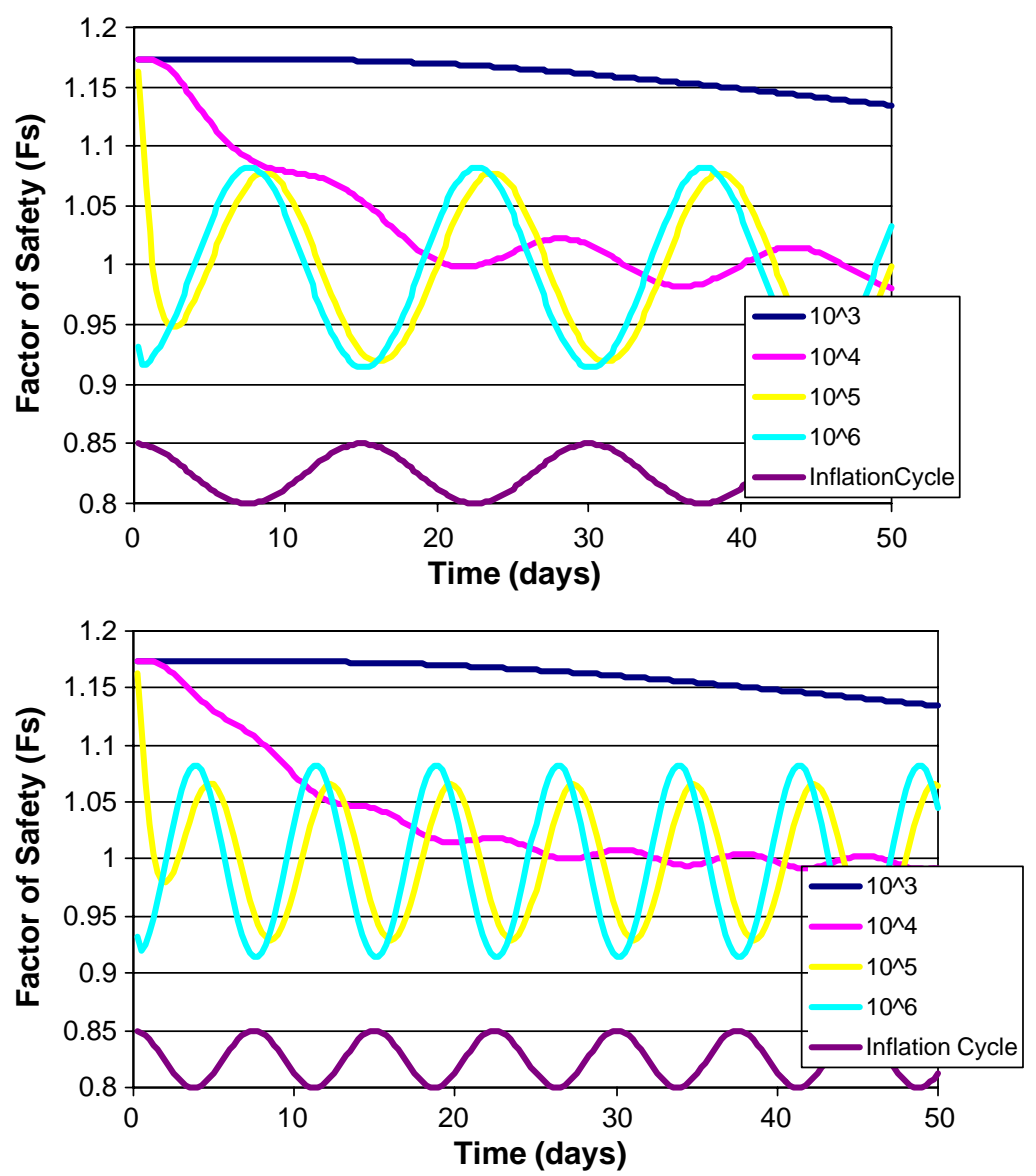

Fig. 6. Variation of Factor of Safety with time for harmonic pressurisation of the interior of Mount St. Helens. Average gas pressure $p_{i}$ is 10 $\mathrm{MPa}$, with a harmonic overpressure of $5 \mathrm{MPa}$. Edifice strength is characterized by $\phi=30^{\circ}$ and $c=1 \mathrm{MPa}$. Gas diffusivities $(\kappa)$ are varied, as shown. Interior inflation cycle is shown at the base of each figure. Top: inflation at 1 cycle per 15 days; Bottom: inflation at 1 cycle per 7.5 days. The pressurisation cycle is synchronous with the response for high diffusivities, with $\kappa>10^{6} \mathrm{~m}^{2} / \mathrm{d}$, but asynchronous for lower magnitudes of diffusivity, with $\kappa<10^{3} \mathrm{~m}^{2} / \mathrm{d}$. 
central pressure conduit is of an assumed radius of $100 \mathrm{~m}$, although the resulting pressure distribution is not overly sensitive to this dimension. From the final blast geometry, a sector angle of $90^{\circ}$ is selected ( $\xi=90^{\circ}$ in Fig. 5). In addition to the geometry, stability is sensitive to the parameters controlling pressurisation and the strength of the volcano flank, in this work set to the-order-of a cohesion of $1 \mathrm{MPa}$, and friction angle of $30^{\circ}$.

Inflation pressures are not well constrained, but for a summit elevation of $1000 \mathrm{~m}$ above the top of the pressurised conduit, hydrostatic and lithostatic limits place total fluid pressures between approximately 10 and $23 \mathrm{MPa}\left(1000 \mathrm{~m} 10 \times 10 \mathrm{kN} / \mathrm{m}^{3}, 1000 \mathrm{~m} \times 23 \mathrm{kN} /\right.$ $\mathrm{m}^{3}$, respectively). This pressure is selected as an unmeasured background pressure close to the hydrostatic, and cycling to a magnitude close to the midpoint between the hydrostatic and the lithostatic. Certainly, peak pressures implicated in large collapses at other andesitic volcanoes have been inferred (from deformation measurements) to have been close to the lithostatic (e.g. for Soufriere Hills volcano on Montserrat; Voight et al., 1999). Thus, the mean conduit pressure is set at $10 \mathrm{MPa}$, with a cycled increment of 5 $\mathrm{MPa}$, added, as $10+5 \cos (2 \pi \omega t)[\mathrm{MPa}]$. The inflation period is recovered from the seismic activity of Fig. $4 \mathrm{a}$ and b, as 1 cycle per 7.5 days, and is shown in Fig. 6 with an inflation period of 1 cycle in 15 days for comparison.

A friction angle of $\phi=30^{\circ}$ and cohesion of $c=1 \mathrm{MPa}$ is capable of producing failure on a detachment dipping at $35^{\circ}$, as estimated from the post-failure geometry. For interior pressurisation of an edifice of varying hydraulic diffusivities ranging from $10^{3} \mathrm{~m}^{2} / \mathrm{d}<\kappa<10^{6}$ $\mathrm{m}^{2} / \mathrm{d}$, the change in stability with time is described in Fig. 6 for inflation periodicities of 7.5 and 15 days. Two suites of behaviour are evident. For high diffusivities, representing high relative permeabilities, the minimum safety factor is phase-offset in time relative to the peak interior pressure - the minimum stability occurs after peak pressure has been applied. As permeabilities increase, the minimum stability is synchronous with maximum pressure, and the edifice is the least stable. As noted in the results shown in Fig. 6, the minimum stability results where the hydraulic diffusivity is the highest, however, the ultimate May 18, 1980 lateral blast was not synchronous with the maximum overpressurisation, as apparent from the time evolution of the seismic scaling exponent $D$ and of the low frequency events and of the harmonic tremor.

For this reason, a plausible mechanism for collapse is that the diffusivity is of intermediate magnitude, of the order of $\kappa=10^{4} \mathrm{~m}^{2} / \mathrm{d}$, with one cycle per 5 days through one cycle per 30 days by steps of 5 days, as

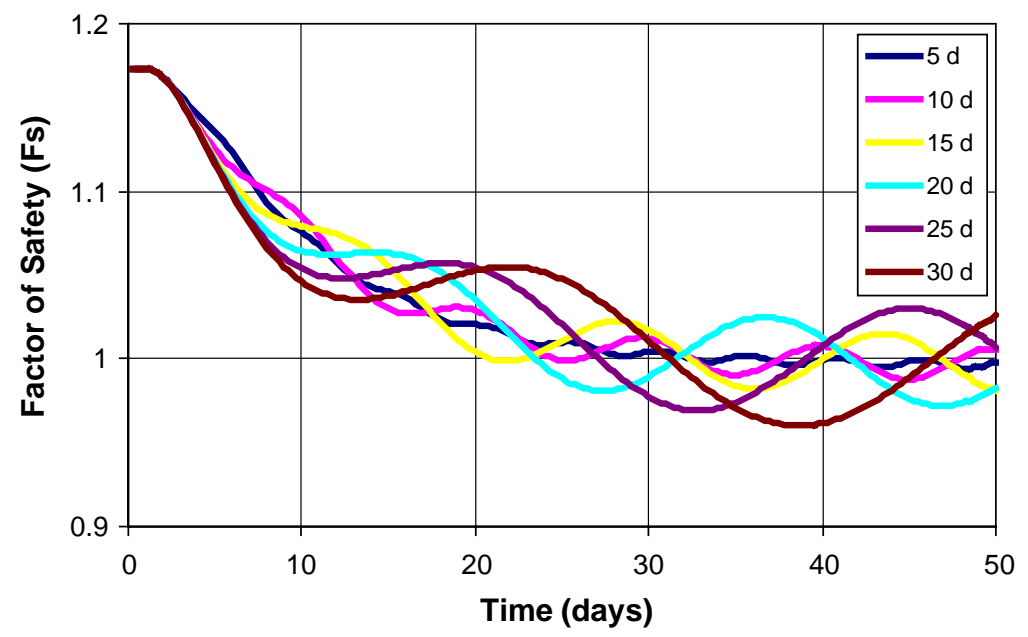

Fig. 7. Variation of Factor of Safety with time for harmonic pressurisation of the interior of Mount St. Helens. Average interior conduit gas pressure $p_{i}$ is $10 \mathrm{MPa}$, with a harmonic overpressure $d p_{i}$ of $5 \mathrm{MPa}$. Edifice strength is characterized by and $c=1 \mathrm{MPa}$. Gas diffusivity $(\kappa)$ is retained at the maximum feasible value (to give the maximum destabilizing influence). Traces represent stability behaviour at cyclic pressurisation periods of one cycle per 5 days, through one cycle per 30 days. 
inferred from the seismic data. This results in monotonically decreasing minimum stabilities (Fig. 7), irrespective of different cyclic pressurisation periods considered, with the minimum stability asynchronous with the peak overpressure, mimicking the collapse behaviour inferred by the seismic record.

The temporary slowing in the rate of change of the seismic scaling exponent $D$ (see Section 3.2 and Fig. 4), apparent from the end of March to early May, paradoxically suggests that the rate of new crack generation slows while the mountain still slowly approaches failure. Consequently, weakening of the edifice primarily results not from its disaggregation, but from penetration of gas overpressures into the flank carapace. This penetration is driven by the cyclic augmentation of gas pressures at the volcano conduit-these gas pressures diffuse outwards, reduce effective stresses, and weaken the flank while driving forces remain essentially unchanged. Thus, failure need not occur on the first cycle of peak pressurisation, but may be delayed over multiple cycles. In this instance, failure occurs both as core pressures are diminishing (post-peak), and following the second cycle of pressurisation - both these observations are consistent with the diffusion of a pressure pulse from the volcano core. Pressures are inferred from the recorded number of low-frequency events, used as a proxy for the unmeasured magnitude of pressurisation.

\section{Conclusions}

Seismicity preceding and accompanying the cataclysmic May 18, 1980 eruption at Mount St. Helens has been analysed in terms of temporal variations of the scaling exponent $D$ inferred from frequencymagnitude statistics of volcano-tectonic events. Variations in the inferred magnitude and rate-of-change of $D$ allow estimation of the evolution of fracture development and advance that accompany the progress towards eruption inside the volcano. An abrupt decrease in $D$ occurs at the end of March 1980 and indicates that major ruptures formed within a few days, but did not lead to the ultimate failure of the edifice. A steady decrease in $D$ persists from the end of March to early May, and suggests the prevalence of a mechanism of slow fracturing that acts on a time scale of several days ( $\sim-10$ days), and involves the development of un-connected cracks that ultimately coalesce, rupture, and lock. This mechanism results in a repetitive pattern of step decreases in $D$, each cycle culminating in the production of a larger rupture that results from the coalescence of smaller cracks. A final decrease in $D$ occurs in early May and is responsible for the persistent pattern of major ruptures that are ultimately implicated in the eruption onset.

Independent measurements of the occurrence of two low frequency earthquake swarms, coupled to high values of harmonic tremor indicate the action of two main cycles of fluid pressurisation on a longer time scale ( $\sim 20-25$ days). If event frequency is used as a proxy for interior pressurisation, then ultimate failure of the edifice occurs both in a period absent significant crack growth, and as interior gas pressurisation is diminishing. These observations are consistent with a model where the flank weakens and ultimately fails as gas pressures build by escape from the core.

The mechanical response of the volcanic edifice is examined in response to harmonic pressurisation by gas exsolved from an internal magma conduit. Cyclic inflation periods of the order of 5 to 30 days are examined, representing periodicities inferred both by the $D$ value and by the low frequency and harmonic tremor, respectively. For this range of inflation periods, the weakening of the flank is delayed relatively to peak pressurisation of the core, and failure may occur as core pressures are diminishing. Similarly, the edifice may withstand multiple prior pulses of pressurisation to the same driving magnitude before critical weakening of the edifice occurs, and failure ensues. Notably, this may occur absent in any degradation in strength of the flank materials via crack formation or rupture, which would necessarily produce a signal in the $D$ value, which is not observed. Rather, failure occurs as fluid pressures build, effective stresses drop, and the flank collapses as resisting forces diminish as the forces driving failure remain the same or increase. Thus, the observed 'delayed failure' is plausible for a mechanism that involves gas transport within the volcano and for reasonable estimates of hydraulic diffusivities of intermediate magnitude - of the order of $\kappa=10^{4} \mathrm{~m} /$ $\mathrm{d}^{2}$. This results in a minimum stability asynchronous 
with the peak overpressure, as inferred from long period seismicity, and congruent with observations of muted rupture immediately prior to collapse, as inferred from the $D$ value. These observations reinforce the important consideration of multiple signals as eruption precursors, in particular where the dominant mechanism may switch in the immediate run-up to eruption.

\section{References}

Aki, K., 1965. Maximum likelihood estimate of $b$ in the formula log $N=a-b M$ and its confidence limits. Bull. Earthq. Res. Inst. Tokyo Univ. 43, 237-239.

Aki, K., 1981. A probabilistic synthesis of precursory phenomena, in earthquake prediction: an international review. In: Simpson, D.W., Richards, P.G. (Eds.), Earthquake Prediction. American Geophysical Union, M. Ewing Series, vol. 4, pp. 566-574.

Anderson, O.L., Grew, P.C., 1977. Stress corrosion theory of crack propagation with applications to geophysics. Rev. Geophys. Space Sci. 15 (1), 77-104.

Carslaw, H.S., Jaeger, J.C., 1959. Conduction of Heat in Solids, (Second Edition). Oxford University Press (510 pp.).

Christiansen, R., Peterson, D., 1981. Chronology of the 1980 eruptive activity. In: Lipman, P.W., Mullineaux, D.R. (Eds.), The 1980 Eruptions of Mount St. Helens, Washington. United States Geological Survey Professional Paper, vol. 1250, pp. $17-30$.

Elsworth, D., Voight, B., 2001. The mechanics of harmonic gas pressurisation and failure of lava domes. Geophys. J. Int. 145, $187-198$

Endo, E.T, Malone, S.D., Noson, L.L., Weaver, C.S., 1981. Locations, magnitudes and statistics of the March 20-May18 earthquake sequence. In: Lipman, P.W., Mullineaux, D.R. (Eds.), The 1980 Eruptions of Mount St. Helens, Washington. United States Geological Survey Professional Paper, vol. 1250, pp. $93-107$.

Gudmundsson, A., Fjeldskaar, I., Brenner, S.L., 2002. Propagation pathways and fluid transport of hydrofractures in jointed and layered rocks in geothermal fields. J. Volcanol. Geotherm. Res. $116,257-278$.

Gutenberg, B., Richter, C.F., 1956. Magnitude and energy of earthquakes. Ann. Geofis. 9 (1), 1-15.

Kanamori, H., Anderson, D.L., 1975. Theoretical bases of some empirical relations in seismology. Bull. Seismol. Soc. Am. 65, $1073-1095$.

Kilburn, C.R.J., 2003. Multiscale fracturing as a key to forecasting volcanic eruptions. J. Volcanol. Geotherm. Res. 2623, 1-19.

Kilburn, C.R.J., Voight, B., 1998. Slow rock fracture as eruption precursor at Soufriere Hills volcano, Montserrat. Geophys. Res. Lett. 25 (19), 3665-3669.

Lipman, P.W., Moore, J.G., Swanson, D.A., 1981. Bulging of the north flank before the May 18 eruption-geodetic data.
In: Lipman, P.W., Mullineaux, D.R. (Eds.), The 1980 Eruptions of Mount St. Helens, Washington. United States Geological Survey Professional Paper, vol. 1250, pp. 143-155.

Lockner, D.A., Byerlee, J.D., Kuksenko, V., Ponomarev, A., Sidorin, A., 1991. Quasi static fault growth and shear fracture energy in granite. Nature 350, 39-42.

Main, I.G., 1991. A modified Griffith criterion for the evolution of damage with a fractal distribution of crack lengths: application to seismic event rates and $b$-values. Geophys. J. Int. 107, 353-362.

Main, I.G., 1992. Damage mechanics with long-range interactions: correlations between the seismic $b$-value and the fractal twopoint correlation dimensions. Geophys. J. Int. 111, 531-541.

Main, I.G., 1999. Applicability of time-to-failure analysis to accelerated strain before earthquakes and volcanic eruptions. Geophys. J. Int. 139, F1-F6.

Main, I.G., Meredith, P.G., 1991. Stress corrosion constitutive laws as a possible mechanism of intermediate-term and short-term seismic quiescence. Geophys. J. Int. 107, 363-372.

Malone, S., 1990. Mount St. Helens, the 1980 re-awakening and continuing seismic activity. Geosci. Can. 17 (3), 146-150.

Mandelbrot, B.B., 1982. The Fractal Geometry of Nature. Freeman, San Francisco, pp. 256-271.

Meredith, P.G., Main, I.G., Jones, C., 1990. Temporal variations in seismicity during quasi-static and dynamic rock failure. Tectonophysics $175,249-268$.

Minakami, T., 1990. Prediction of volcanic eruptions. In: Civetta, L., Gasparini, P., Luongo, G., Rapolla, A. (Eds.), Physical Volcanology. Elsevier, Amsterdam, pp. 1-27.

Scandone, R., Malone, S.D., 1985. Magma supply, magma discharge and readjustment of the feeding system of Mt. St. Helens during 1980. J. Volcanol. Geotherm. Res. 23, 239-262.

Shaw, H.R., 1980. The fracture mechanisms of magma rise from mantle to the surface. In: Hargraves, R.B. (Ed.), Physics of Magmatic Processes. Princeton University Press, Princeton, NJ, pp. $201-264$.

Simmons, J., Elsworth, D., Voight, B., 2004. Stability of exogenous lava lobes during intense precipitation. Bull. Volcanol 66, 725-734.

Takeo, M., 1983. Source mechanisms of Usu volcano, Japan, earthquakes and tectonic implications. Phys. Earth Planet. Inter. $32,241-264$.

Tinti, S., Mulargia, F., 1985. An improved method for the analysis of the completeness of a seismic catalogue. Lett. Nuovo Cimento 42, 21-27.

Turcotte, D.L., 1989. Fractals in geology and geophysics. PAGEOPH 131, 171-196.

Vinciguerra, S., 1999. Scaling exponents as a tool in detecting stress corrosion crack growth leading to the September-October 1989 flank eruption at Mt. Etna volcano. Geophys. Res. Lett. 26 (24), $3685-3688$.

Vinciguerra, S., 2002. Damage mechanics preceding the September-October 1989 flank eruption at Mt. Etna volcano inferred by seismic scaling exponents. J. Volcanol. Geotherm. Res. 113, $391-397$.

Voight, B., Elsworth, D., 2000. Instability and collapse of lava domes. Geophys. Res. Lett. 27 (1), 1-4. 
Voight, B., Janda, R.J., Glicken, H., Douglass, P.M., 1983. Nature and mechanics of the Mount St. Helens rockslide-avalanche of 18 May 1980. Geotechnique 33, 243-273.

Voight, B., Sparks, R.S.J., Miller, A.D., Stewart, R.C., Hoblitt, R.P., Clarke, A., Ewart, J., Aspinall, W., Baptie, B., Druitt, T.H., Herd, R., Jackson, P., Lockhart, A.B., Loughlin, S.C., Lynch, L.,
McMahon, J., Norton, G.E., Robertson, R., Watson, I.M., Young, S.R., 1999. Magma flow instability and cyclic activity at Soufriere Hills Volcano, Montserrat, BWI. Science 283, 1138-1142.

Wyss, M., Shimazaki, K., Wiemer, S., 1997. Mapping active magma chambers by b-value beneath the off-Ito volcano, Japan. J. Geophys. Res. 102, 20413-20422. 